\title{
Poliarterite Nodosa Cutânea na Infância com Gangrena Digital e Possível Associação com o Estreptococo Beta-Hemolítico do Grupo A: Relato de Caso e Revisão de Literatura
}

\section{Cutaneous Polyarteritis Nodosa in Children Presenting with Digital Gangrene and Possible Association with Group A Beta Hemolytic Streptococcus Infection}

\author{
Karina Silva Marins $^{(1)}$, Ronaldo Baptista ${ }^{(2)}$, Vladimir Nentzinsky ${ }^{(3)}$, \\ Mário Newton Leitão de Azevedo(2), Blanca Elena Rios Gomes Bica ${ }^{(2)}$
}

\section{RESUMO}

A poliarterite nodosa cutânea (PAN cutânea) é uma forma particular de vasculite envolvendo artérias de pequeno e médio calibres. A doença pode ser diferenciada da poliarterite nodosa sistêmica pela sua limitação à pele e ausência de envolvimento visceral. Manifesta-se habitualmente por nódulos subcutâneos, livedo reticular e ulceração cutânea. Artralgia, mialgia, neuropatia e sintomas constitucionais, como febre e mal-estar, podem estar presentes. O diagnóstico é, por vezes, de difícil realização, baseando-se na forte suspeita clínica e no exame histológico da pele. Neste trabalho é descrito um caso de PAN cutânea em uma criança de 9 anos com alteração isquêmica grave da ponta dos dedos e nódulos subcutâneos como manifestações iniciais e a provável associação com o estreptococo beta-hemolítico grupo A (EBHGA). A revisão de literatura revela que PAN cutânea na infância está freqüentemente associada à infecção estreptocócica e que atenção especial deveria ser dada a crianças com menos de 10 anos de idade pelo alto risco relatado de auto-amputações.

Palavras-chave: poliarterite nodosa cutânea, vasculite, gangrena, estreptococo beta-hemolítico do grupo A.

\section{INTRODUÇÃO}

A poliarterite nodosa cutânea (PAN cutânea) foi descrita pela primeira vez em 1931 e, desde então, mais de 100 casos já foram relatados ${ }^{(1)}$. É uma patologia rara na faixa etária pediátrica, caracterizada pela presença de vasculite necrosante de vasos de pequeno e médio calibres ${ }^{(2)}$.

\begin{abstract}
Cutaneous polyarteritis nodosa (cutaneous PAN) is a particular form of vasculitis involving small and medium sized arteries. The disease can be differentiated from classic PAN by its limitation to the skin and lack of progression to visceral involvement. Usually it manifests with subcutaneous nodule, livedo reticularis and cutaneous ulceration. Arthralgia, myalgia, neuropathy and constitutional symptoms such as fever and malaise may also be present. Sometimes the diagnosis is difficult, being just based on clinical suspicion and skin histologic exam. In this paper the authors describe the case of cutaneous PAN in a 9 years old child with severe ischemic changes in the fingertips, and subcutaneous nodules as initial signs of cutaneous PAN and probable association with the group A beta hemolytic streptococcus (GABHS). The review of the literature shows that the cutaneous PAN in the childhood may be associated to streptococcal infection and, for this reason, a special care should be given for those under 10 years old due to the high risk of self amputation.
\end{abstract}

Keywords: cutaneous polyarteritis nodosa, vasculitis, gangrene, group A beta hemolytic streptococci.

A doença pode ser diferenciada da poliarterite nodosa clássica pela sua limitação à pele e pela ausência de progressão para envolvimento visceral ${ }^{(3)}$. As manifestações características são nódulo subcutâneo, livedo reticular e ulceração, localizados predominantemente em membros inferiores ${ }^{(3)}$. A idade de início é variável, sendo os dois sexos igualmente atingidos ${ }^{(4)}$. A patogênese é desconhecida ${ }^{(5)}$,

Recebido em 11/6/2007. Aprovado, após revisão, em 13/9/2007. Declaramos a inexistência de conflitos de interesse.

Serviço de Reumatologia do Hospital Universitário Clementino Fraga Filho da Universidade Federal do Rio de Janeiro

1. Médica pós-graduanda em Reumatologia na Universidade Federal do Rio de Janeiro (UFRJ).

2. Professor adjunto do Serviço de Reumatologia do Hospital Universitário Clementino Fraga Filho (HUCFF) UFRJ

3. Médico do Serviço de Reumatologia do HUCFF/UFRJ.

Endereço para correspondência: Karina Silva Marins, Rua Pres. Roosevelt, 98/301, Vila Zelia, 12606-290, Lorena, SP, e-mail:karinasilvamarins@yahoo.com.br 
entretanto supõe-se que mecanismos imunomediados estejam envolvidos. Vários fatores têm sido implicados, incluindo drogas, tuberculose, hepatite e infecções estreptocócicas $^{(3)}$. A doença apresenta um curso benigno e crônico que exibe tendência a recidivas e remissões ${ }^{(3)}$.

O objetivo deste trabalho é apresentar um caso de PAN cutânea na infância acompanhado no Hospital Universitário Clementino Fraga Filho da Universidade Federal do Rio de Janeiro (HUCFF/UFRJ) que apresentou necrose dos dedos como manifestação inicial e sua provável associação com infecção estreptocócica, além de revisão de literatura.

\section{RELATO DO CASO}

G.S.A., sexo feminino, 21 anos, raça caucasiana, natural da Paraíba (RN) e residente no Rio de Janeiro, estudante. Foi admitida no HUCFF apresentando lesões eritemato violáceas dolorosas, nodulares, em região lateral esquerda do abdômen e coxa esquerda, partes anterior e lateral.

Paciente relatava início do quadro aos 9 anos de idade, quando foi picada por uma abelha em segundo quirodáctilo esquerdo e houve o aparecimento de eritema, rubor e prurido local que evoluíram em 24 horas para cianose, inclusive das outras polpas digitais.

Nessa época apresentou muita dor articular e febre concomitante. Após sete dias do início dos sintomas procurou atendimento médico e foi internada. Permaneceu internada por alguns dias, recebendo antibiótico venoso sem êxito, apresentando amputação espontânea do segundo quirodáctilo esquerdo na altura da base.

Recebeu alta hospitalar, apesar de apresentar cianose de vários quirodáctilos de ambas as mãos. Durante os seis meses subseqüentes apresentou dor e cianose que evoluíram para auto-amputação de terceiro e quinto quirodáctilos esquerdo e segundo e quinto quirodáctilos direito, além de reabsorção da falange distal do quarto quirodáctilo direito. Permaneceu assintomática até início de 2004 quando apareceram as lesões em abdômen e coxa e a paciente foi encaminhada ao HUCFF para investigação.

No exame objetivo à admissão, apresentava-se em bom estado geral. A pele apresentava lesões cutâneas eritematovioláceas dolorosas, dispersas em região lateral esquerda de abdômen e parte anterior e lateral de coxa esquerda, com padrão de livedo reticularis. Apresentava nódulos quentes, dolorosos, agrupados em abdômen, coxa e região tibial anterior somente à esquerda. Não havia outras alterações significantes no exame físico.

Dos antecedentes pessoais referia amigdalites de repetição. Não fumava e não havia história de fenômeno de Raynaud, flebite ou perda fetal.

Os antecedentes familiares eram irrelevantes, não se conhecendo outros casos de lesões cutâneas semelhantes.

Os testes laboratoriais mostraram: hemoglobina de 14,4 $\mathrm{g} / \mathrm{dl}$, hematócrito de $42,2 \%$, leucócitos: 8.100 células $/ \mathrm{mm}^{3}$ com diferencial normal e 226.000 plaquetas $/ \mathrm{mm}^{3}$. VHS: $50 \mathrm{~mm}$ na primeira hora. Função renal e hepática normais. Antiestreptolisina O (ASO) de $800 \mathrm{UI} / \mathrm{ml}$ (normal 0-200). As sorologias para hepatite B e C, HIV 1 e 2 foram negativas. As hemoculturas foram igualmente negativas. $\mathrm{O}$ estudo imunológico (FAN, anti-DNA, anti-RNP, ANCA, VDRL e anticardiolipinas) não mostrou alterações.

A radiografia do tórax, o ecocardiograma e a ultrassonografia abdominal não mostraram alterações morfológicas ou patológicas.

A biópsia do nódulo subcutâneo na coxa esquerda mostrou vasculite necrosante de pequenos vasos da derme profunda.

Diante dos dados clínicos e laboratoriais admitiu-se a hipótese de PAN cutânea, confirmada pela histologia, com provável associação neste último episódio com o estreptococo beta-hemolítico do grupo A.

Iniciada a terapêutica com prednisolona $50 \mathrm{mg} / \mathrm{dia}$ e penicilina benzatina 1.200.000 UI, a paciente evoluiu com regressão progressiva de todo o quadro clínico.

Desde a alta hospitalar, a paciente encontra-se assintomática, mantendo-se atualmente apenas em uso de profilaxia com penicilina benzatina 1.200.000 UI de 21/21 dias.

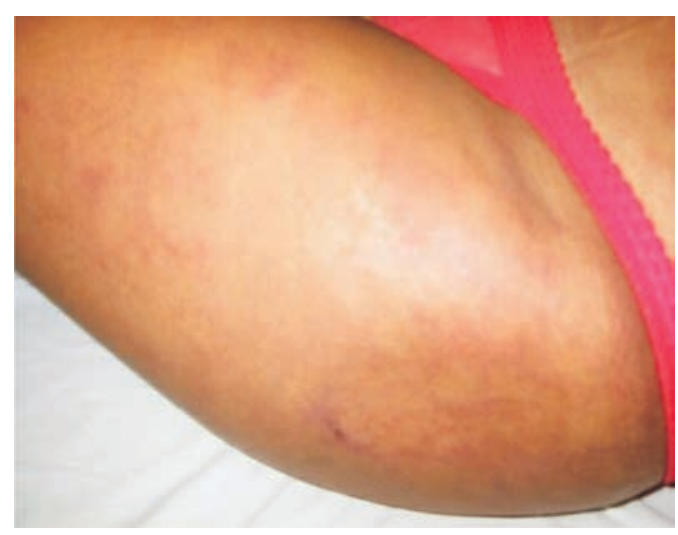

Figura 1 - Coxa esquerda mostrando aspecto de livedo reticular e lesões nodulares eritematosas. 


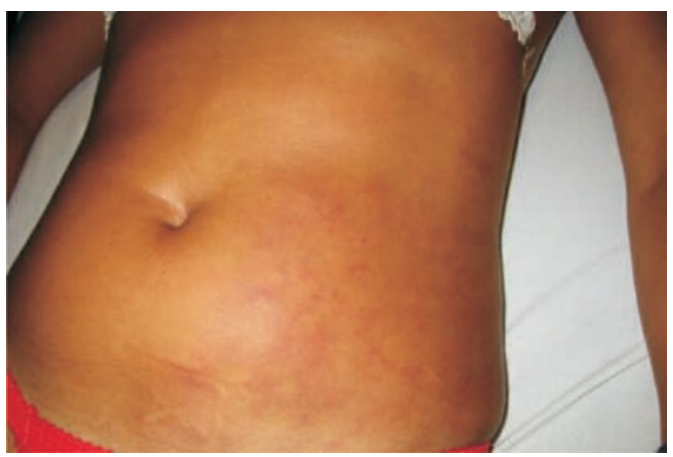

Figura 2 - Abdômen, mostrando em região lateral esquerda a lesão sugestiva de PAN cutânea.

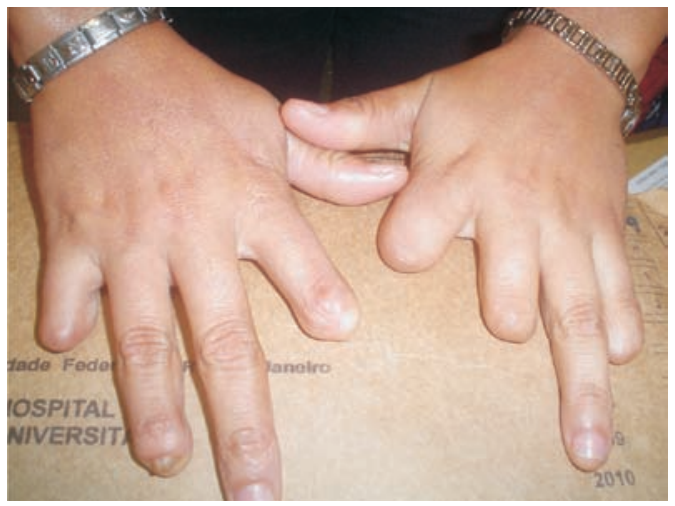

Figura 3 - Mãos direita e esquerda mostrando as seqüelas dos surtos anteriores com amputações de vários quirodáctilos.

\section{DISCUSSÃO E REVISÃO DA LITERATURA}

A PAN cutânea constitui uma forma particular de vasculite, envolvendo normalmente vasos de pequeno e médio calibres, distinguindo-se da forma sistêmica pela ausência de envolvimento visceral e pela evolução benigna, mas crônica recidivante ${ }^{(4,2)}$. Apesar de considerada um processo de doença localizado que poupa artérias viscerais, a doença não é rigorosamente confinada à pele, podendo ter manifestações extracutâneas, como febre, neuropatia regional, miopatia, artralgias e artrite ${ }^{(5,6)}$.

O diagnóstico de PAN cutânea foi estabelecido tendo como base o envolvimento cutâneo predominante, a ausência de envolvimento sistêmico e a biópsia de pele mostrando vasculite necrosante de pequenas artérias da derme profunda. Poliarterite nodosa cutânea na criança deve ser suspeitada na presença de febre, nódulos subcutâneos, livedo reticular e $\operatorname{artralgia}^{(7)}$. Essas lesões não estavam, no entanto, presentes no início do quadro.

A apresentação inicial se deu por meio de gangrena periférica dos dedos das mãos, levando à auto-amputação.
A gangrena periférica desta gravidade resultando autoamputação é incomum nos relatos mais precoces de PAN cutânea $^{(8)}$. Entretanto, a auto-amputação da ponta dos dedos dos pés e das mãos é observada, segundo revisão de literatura, mais freqüentemente em pacientes com menos de 10 anos de idade ${ }^{(8)}$. Essa observação é confirmada pelo caso clínico descrito, no qual a paciente em questão apresentou amputações espontâneas dos dedos das mãos, como manifestação inicial da doença, aos 9 anos de idade. Manifestações iniciais raras, como necrose dos dedos no presente caso, deveriam também ser consideradas um sinal clínico da PAN cutânea ${ }^{(9)}$. A extensão da gangrena e a autoamputação poderiam provavelmente ter sido minimizadas se essa paciente tivesse recebido terapia apropriada em um estágio mais precoce de sua doença ${ }^{(8)}$. A arteriografia dos pés e das mãos pode ser útil, revelando arteriopatia distal com estenose dos vasos ou a ausência de enchimento destes, identificando esses pacientes oportunos para um regime de tratamento agressivo ${ }^{(8)}$.

Os sinais clínicos da maioria dos casos de PAN cutânea foram vistos em nosso paciente: a ausência de progressão para envolvimento sistêmico, recidiva, períodos prolongados de remissão, boa resposta aos corticosteróides e bom seguimento a longo prazo $^{(10)}$.

Antecedentes de infecção estreptocócica têm sido implicados em ambas as formas cutânea e sistêmica da poliarterite nodosa ${ }^{(10)}$. Kumar et al. ${ }^{(8)}$ descreveram a maior série de crianças com PAN cutânea em 1995. Eles observaram possível associação entre PAN cutânea e picada de vespa em uma criança, semelhante ao caso relatado neste trabalho, no qual a paciente apresentou início do quadro após a picada de uma abelha. Tanto a picada da abelha quanto o passado de amigdalites de repetição, com a presença de títulos elevados de ASO, podem ser relacionados como fatores etiológicos desencadeantes da doença. Ainda que a evidência de infecção estreptocócica como um fator etiológico da PAN cutânea em nossa paciente esteja presente claramente, cautela deve ser exercida quanto à interpretação isolada de elevações nos marcadores sorológicos das infecções estreptocócicas na ausência de culturas documentadas ou uma apresentação clínica apropriada sugestiva de infecção estreptocócica ${ }^{(10)}$. Nossa paciente relatava episódios de faringoamigdalite precedendo o aparecimento das lesões cutâneas dolorosas no abdômen em várias ocasiões. Albornoz et al. ${ }^{(10)}$ relatam em seu estudo que elevação de imunoglobulina policlonal sozinha poderia justificar títulos elevados de ASO. Verbov ${ }^{(11)}$ relatou um paciente com PAN cutânea, no qual títulos de 
ASO foram elevados em múltiplas ocasiões na ausência de culturas de orofaringe positiva e sintomas clínicos.

A pesquisa por um agente infeccioso é importante quando uma vasculite é diagnosticada, em particular a poliarterite nodosa ${ }^{(12)}$. Em crianças com PAN cutânea, a avaliação deve incluir estudos laboratoriais para detecção de infecção estreptocócica ${ }^{(13)}$ e a indicação de profilaxia secundária com penicilina benzatina parece ser adequada, em especial para as crianças com formas graves e/ou recorrências da doença ${ }^{(2)}$.

As síndromes vasculíticas são o resultado de inflamação e necrose das paredes do vaso sanguíneo que podem resultar de envolvimento primário ou podem ocorrer como uma das características de outras doenças bem definidas (lúpus eritematoso sistêmico, artrite reumatóide $)^{(8)}$.

Diaz-Perez e Winkelman ${ }^{(14)}$ descrevem a relação entre poliarterite nodosa clássica e PAN cutânea como semelhante à relação entre lúpus eritematoso e lúpus discóide, na qual as manifestações deste último são a pele em $95 \%$ dos casos.

Em 1931, Lindberg ${ }^{(15)}$ descreveu a existência da variante cutânea da PAN, sem envolvimento visceral e com um prognóstico mais favorável. Desde então, sua existência como uma entidade de doença distinta tem sido motivo de debate ${ }^{(4,6,9,16)}$. Entretanto, estudos recentes de seguimento em longo prazo têm mostrado, claramente, que PAN cutânea raramente progride para a forma sistêmica ${ }^{(9)}$. O conceito de poliarterite cutânea foi reforçado pela descrição de 52 pacientes observados ao longo de 27 anos, que não tiveram evidência clínica de envolvimento sistêmico ${ }^{(6)}$.

A idade de início é variável ${ }^{(4)}$. Fathalla et al.$^{(17)}$ relatam em seu estudo que a PAN cutânea em criança é mais comum na idade escolar. Os dois sexos são igualmente atingidos, contrariamente à predominância masculina da PAN sistêmica $^{(4)}$. A prevalência da doença não está estabelecida, mas não é particularmente rara, mais de 250 casos foram descritos na literatura até $1999^{(4-6)}$. Não há consenso sobre o número total de casos pediátricos de PAN cutânea na literatura $^{(17)}$. Em 1998, Ginrate, Pereiro e Toribio notaram o total de 45 casos pediátricos de PAN cutânea relatados na literatura, datados desde 1965, enquanto Mocan et al. identificaram somente 24 casos relatados em sua revisão de literatura, datados desde $1977^{(17)}$.

O quadro clínico da PAN cutânea apresenta como manifestação característica em ordem decrescente de freqüência: nódulos cutâneos e/ou subcutâneos, livedo reticular, ulcerações e gangrena ${ }^{(2,6)}$.
Os nódulos característicos da PAN cutânea ${ }^{(1)}$ são normalmente dolorosos e tendem a ocorrer em grupos, medindo de 0,5 a $3 \mathrm{~cm}$ de diâmetro ${ }^{(6)}$. Freqüentemente, no primeiro sinal da doença, os nódulos são predominantemente localizados nos membros inferiores, abaixo dos joelhos, acompanham o trajeto vascular e estão presentes em $55 \%$ a $70 \%$ dos casos em crianças e em até $95 \%$ dos adultos, podendo envolver qualquer parte do corpo, exceto superfícies mucosas ${ }^{(2,6)}$. Em contraste, as lesões cutâneas típicas na PAN sistêmica são geralmente púrpura palpável, sendo a presença de nódulos, livedo, úlceras, necrose ou gangrena $\operatorname{rara}^{(16)}$.

Livedo reticular normalmente ocorre após o aparecimento dos nódulos e é limitado às áreas de nodulação. Este modelo de mancha com livedo reticular e nódulos inflamatórios dentro das ramificações tem sido considerado uma característica dermatológica única da PAN cutânea e está descrita como uma lesão patognomônica ${ }^{(6)}$. Ocorre em cerca de $40 \%$ a $45 \%$ dos casos e pode persistir por períodos prolongados, fato observado no caso relatado e não associado à atividade da doença ${ }^{(2)}$.

Referidas em $40 \%$ dos adultos, as úlceras e as gangrenas não são freqüentes na PAN cutânea da infância, sendo relatadas em 7,5\% das séries da literatura ${ }^{(2)}$, apesar de Kiss et al. terem observado em $54 \%$ dos seus pacientes ${ }^{(2)}$. Tal achado também foi observado em nossa paciente que apresentou gangrena com perda das falanges distais. Essa freqüência elevada poderia sugerir maior gravidade da doença e/ou demora no diagnóstico. A auto-amputação da ponta dos dedos dos pés e das mãos ocorre mais freqüentemente em crianças com menos de $10 \operatorname{anos}^{(17)}$.

Muitos casos de PAN cutânea têm sido descritos na Coréia, segundo os estudos de Yoon e Lee ${ }^{(18)}$ e Bang et $a l .{ }^{(19)}$, sendo que nove deles apresentaram-se com gangrena digital, mas necrose dos dedos, como neste caso, é um achado muito raro $^{(3)}$.

As manifestações extracutâneas são documentadas em uma porcentagem significante de casos. Sintomas neuromusculares são normalmente localizados nas áreas de envolvimento da pele. São comuns: dores fortes e rigidez nas panturrilhas, por causa da miosite, e, também, os sintomas neurológicos periféricos, os quais incluem torpor, parestesias, neurites dolorosas e mononeurite multiplex ${ }^{(6)}$. Artrite não-erosiva e/ou artralgia também são freqüentes, ocorrendo em $70 \%$ a $90 \%$ dos casos, com características migratórias e comprometimento preferencial de grandes articulações de membros inferiores ${ }^{(2,6)}$. Febre, acompanhan- 
do o surto inicial ou as recidivas, é comum, ocorrendo em $80 \%$ dos casos referidos na literatura ${ }^{(2)}$.

O diagnóstico é confirmado pela biópsia incisional, que deve mostrar a derme profunda e o tecido subcutâneo. A inflamação das artérias de pequeno e médio calibres é histologicamente idêntica ao visto nas lesões viscerais da PAN sistêmica e ocorre na junção derme panicular ${ }^{(2,6)}$. Os achados histopatológicos podem ser divididos em quatro estágios: estágio degenerativo com degeneração da parede arterial e deposição de material fibrinóide e destruição parcial ou completa da lâmina elástica externa e interna; estágio inflamatório agudo com um infiltrado geralmente composto de neutrófilos com alguns eosinófilos ao redor e dentro da parede arterial; estágio de tecido de granulação com um infiltrado também contendo linfócitos e macrófagos e proliferação da íntima e trombose com oclusão do lúmen levando à ulceração; e estágio final com proliferação fibroblástica estendendo-se na área perivascular ${ }^{(3,16)}$. A vasculite leucocitoclástica pode coexistir na amostra da biópsia em mais de $30 \%$ desses indivíduos ${ }^{(5)}$. Em relação aos achados da imunofluorescência direta, a presença de IgM ou depósito de C3 nas paredes dos vasos têm sido encontradas em alguns pacientes, não somente na artéria profunda envolvida mas também na superfície e em pequenos vasos não envolvidos ${ }^{(16)}$.

Uma velocidade de hemossedimentação (VHS) elevada é a única anormalidade laboratorial consistente, ainda que anemia, leucocitose e trombocitose tenham sido relatadas, enquanto outros marcadores sorológicos e imunológicos não são achados freqüentes ${ }^{(3,5,6)}$. É interessante ressaltar que a VHS é um parâmetro bastante útil para a monitorização da resposta terapêutica, porém pode ser um dos últimos parâmetros laboratoriais a se normalizar e não necessariamente relacionado à atividade clínica da doença ${ }^{(2)}$.

A etiologia da PAN cutânea é desconhecida, mas depósito de $\operatorname{IgM}$, C3 e fibrina dentro dos vasos afetados na imunofluorescência direta sugerem que complexos imunes estejam envolvidos na patogênese da doença, entretanto complexos imunes circulantes têm sido detectados com pouca freqüência ${ }^{(6)}$. Vários fatores têm sido implicados como agentes desencadeantes no desenvolvimento da PAN cutânea e incluem, entre outros, doença inflamatória intestinal, tuberculose, hepatite B e infecções estreptocócicas $^{(3,10)}$.

Muitos casos de vasculite necrosante são resultado de mecanismos imunopatogênicos, vistos em decorrência de reações de hipersensibilidade por antígenos conhecidos ou desconhecidos ${ }^{(8)}$. Kumar et al. ${ }^{(8)}$ descreveram uma das maiores séries de crianças com PAN cutânea em $1995^{(17)}$. Eles analisaram 10 crianças e relataram associação com o estreptococo beta-hemolítico do grupo A (EBHGA) em duas, com drogas em duas, com a vacina tríplice (difteria, tétano, coqueluche) em uma, com malária em outra paciente e com picada de vespa em um outro caso ${ }^{(2,8)}$. Outros relatos têm enfatizado o papel de infecções estreptocócicas, como o relato de Fink, em 1991 ${ }^{(20)}$, e o relato de Till e Amos, em $1997^{(21)}$. Kiss et al. ${ }^{(2)}$ relataram sete de dez crianças (70\%) com PAN cutânea que apresentaram títulos elevados de antiestreptolisina $\mathrm{O}$ (ASLO), com valores que variaram de 500 a 6.250 U Todd, sugerindo infecção estreptocócica recente ${ }^{(2)}$.

Uma porcentagem importante de pacientes com a forma cutânea e mesmo com a forma sistêmica de PAN apresentam títulos de ASLO elevados. A possibilidade de o EBHGA estar envolvido na etiologia da PAN cutânea já foi discutida em várias publicações na literatura ${ }^{(8,22)}$, sugerindo-se que, a exemplo da febre reumática, a PAN cutânea poderia ser considerada uma complicação pós-estreptocócica ${ }^{(2)}$. É interessante ressaltar que a maior freqüência das publicações sobre PAN cutânea na infância acompanha temporalmente o reaparecimento de cepas estreptocócicas densamente encapsuladas e com alta virulência que justificaram, pelo menos parcialmente, as descrições da síndrome do choque tóxico associada ao EBHGA e o recrudescimento da febre reumática, observada em vários países a partir de 1985 e traduzida pelo aumento do número de casos da doença e pela maior gravidade de suas manifestações clínicas. Esse aumento no número de casos de PAN cutânea parece ser proveniente, em especial, de casuísticas de países como a Índia, onde as complicações pós-estreptocócicas, como a febre reumática, ainda constituem importantes problemas de saúde ${ }^{(2)}$. Kiss et al..$^{(2)}$ acrescentam ainda que os resultados observados por eles e os resultados das várias séries da literatura sugerem participação etiológica importante do EBHGA na PAN cutânea da infância, sendo possível que esta correlação seja ainda mais estreita, especialmente quando se considera que a sensibilidade da ASLO para o diagnóstico da infecção estreptocócica recente não ultrapassa valores de $80 \%$ para infecções de orofaringe e de $60 \%$ para as infecções de pele e que este é, praticamente, o único exame utilizado nas várias séries para o diagnóstico de infecção estreptocócica ${ }^{(2)}$.

A evolução da PAN cutânea é habitualmente benigna, contrariamente à da PAN sistêmica, que geralmente é mortal em dois anos na ausência de tratamento ${ }^{(4)}$. A poliarterite cutânea tem um curso variável com freqüentes recidivas e 
remissão após terapêutica sintomática ou espontânea, dificultando a avaliação do tratamento ${ }^{(6,8)}$. As manifestações extracutâneas podem surgir até 20 anos após o aparecimento dos nódulos, o que implica seguimento prolongado deste tipo de doente ${ }^{(4)}$. É muito importante avaliar esses pacientes cuidadosamente para excluir envolvimento sistêmico, não somente na primeira consulta, mas, também, na continuidade do seguimento ${ }^{(16)}$.

O diagnóstico diferencial com as outras vasculites, assim como com a PAN sistêmica, nem sempre é fácil. A presença de nódulos cutâneos ocorre em outras vasculites, como na doença de Churg-Strauss $(30 \%)^{(4,23)}$, na PAN sistêmica $(28 \%)^{(4,23)}$, na doença de Wegener $(14 \%)^{(4,23)}$, na doença de Takayasu $(10 \%)^{(4,23)}$ e também na artrite reumatóide ${ }^{(4,23)}$

Apesar de não haver séries prospectivas placebo-controladas, duplo cego, para avaliar a terapia, o prognóstico benigno da PAN cutânea justifica o tratamento conservador que poderia ser iniciado com baixas doses de sulfassalazina, antiinflamatórios não-esteróides, colchicina, dapsona, pentoxifilina ou salicilato, que podem induzir remissão ou aliviar os sintomas ou sinais da doença ${ }^{(4,8)}$. Os imunossupressores são usados somente nas formas mais graves ${ }^{(4)}$. O tratamento com antibiótico pode ser necessário em pacientes com infecções estreptocócicas ou outras infecções documentadas. Se não houver resposta, doses baixas ou moderadas de corticóides e/ou metotrexato semanalmente

\section{REFERÊNCIAS}

1. Fitzgerald DA, Verbov JL: Cutaneous polyarteritis nodosa. Arch Dis Child 74(4): 367, 1996.

2. Kiss MHB, Silva CHM, Silva CAA, Janz LL: Poliarterite nodosa cutânea na infância: relato de 11 casos e possível associação com o estreptococo beta-hemolítico do grupo A. Rev Bras Reumatol 38(6): 373-7, 1998.

3. Choi SW, Lew S, Cho SD, et al.: Cutaneous polyarteritis nodosa presented with digital gangrene: a case report. J Korean Med Sci 21: 71-3, 2006.

4. Camões AC, Marques S, Pacheco MH, Malhado J: Periarterite nodosa cutânea: um caso clínico. Rev Soc Port Med Int 12(2): 63-6, 2005.

5. Kelley WN, Harris ED, Ruddy S, Sledge CB. Textbook of rheumatology. 5.ed. Philadelphia: W. B. Saunders Company; 1997. $2 \mathrm{v}$.

6. Koopman WJ: Arthritis and allied conditions: a textbook of rheumatology. 13.ed. USA: Williams \& Wilkins - a waverly company; 1997. $2 \mathrm{v}$

7. Assicot C, Bourrat E, Prigent F, et al.: Cutaneous polyarteritis nodosa in children: three cases. Ann Dermatol Venereol 129(2): 207-11, 2002. têm sido usadas com boa resposta ${ }^{(4,16)}$. Stussi et al..$^{(9)}$ descreveram um caso de PAN cutânea com necrose digital que respondeu à terapia com prostaglandina $\mathrm{I} 2$, bloqueadores de canais de cálcio e corticosteróide ${ }^{(3)}$. A associação com infecções estreptocócicas tem sido enfatizada com vários graus de eficácia da profilaxia com penicilina ${ }^{(17)}$. A amigdalectomia pode ser recomendada como um adjuvante ao corticóide nos casos de PAN cutânea com amigdalite e/ou infecção estreptocócica crônicas ${ }^{(24)}$. Na criança com PAN cutânea, a avaliação deveria incluir estudos laboratoriais para detectar infecção estreptocócica ${ }^{(13)}$, e atenção especial deveria ser dada a crianças com menos de 10 anos de idade pelo alto risco relatado de auto-amputações ${ }^{(17)}$.

\section{CONCLUSÃO}

Toda criança com diagnóstico de PAN cutânea deve ser avaliada para possível associação com infecção estreptocócica, e o tratamento com antibiótico deve ser indicado quando confirmada a existência de infecção. A profilaxia com penicilina benzatina parece ser eficaz. Amigdalectomia pode ser recomendada como um adjuvante ao corticosteróide para os casos de PAN cutânea com amigdalite crônica. Atenção especial deveria ser dada para crianças com menos de 10 anos de idade, com a instituição do tratamento precocemente pelo alto risco de auto-amputações.

8. Kumar L, Thapa BR, Sarkar B, Walia BN: Benign cutaneous polyarteritis nodosa in children below 10 years of age: a clinical experience. Ann Rheum Dis 54(2): 134-6, 1995.

9. Stussi G, Schneider E, Trueb RM, Seebach JD: Acral necrosis of the fingers as initial manifestation of cutaneous polyarteritis nodosa: a case report. Angiology 52(1): 63-7, 2001.

10. Verbov J: Cutaneous polyarteritis nodosa in a young child. Arch Dis Child 55(7): 569-72, 1980.

11. Albornoz MA, Benedetto AV, Korman M, Mcfall S, Tourtellotte CD, Myers AR: Relapsing cutaneous polyarteritis nodosa associated with streptococcal infections. Int J Dermatol 37(9): 664-6, 1998.

12. Leveque L, Turcu A, Bielefeld P, et al.: Adult cutaneous periarteritis nodosa and dental infections: role of streptococci. Rev Med Interne 22(10): 992-6, 2001.

13. Sheth AP, Olson JC, Esterly NB: Cutaneous polyarteritis nodosa of childhood. J Am Acad Dermatol 31(4): 561-6, 1994.

14. Diaz-Perez JL, Winkleman RK: Cutaneous polyarteritis nodosa. Arch Dermatol 110: 407-14, 1974.

15. Lindberg K: Ein beitrag zur kenntnis der periarteritis nodosa. Acta Med Scand 76: 183-225, 1931

16. Bauza A, Espana A, Idoate M: Cutaneous polyarteritis nodosa. Br J Dermatol 146(4): 694-9, 2002. 
17. Fathalla BM, Miller L, Brady S, Schaller JG: Cutaneous polyarteritis nodosa in children. J Am Acad Dermatol 53(4): 724-8, 2005.

18. Yoon $\mathrm{CH}$, Lee CW: Clinical patterns of cutaneous lesions on the legs in patients with cutaneous polyarteritis nodosa. Korean J Dermatol 41: 869-72, 2003.

19. Bang KT, Herr H, Kim MJ, et al.: Three cases with cutaneous polyarteritis nodosa. J Korean Rheum Assoc 11: 447-52, 2004.

20. Fink C: The role of the streptococcus in post-streptococcal reactive arthritis and childhood polyarteritis nodosa. J Rheumatol 18: 14-20, 1991.
21. Till SH, Amos RS: Long term follow-up of juvenile onset cutaneous polyarteritis nodosa associated with streptococcal infection. Br J Rheumatol 36(8): 909-11, 1997.

22. Dillon MJ, Ansell B: Vasculitis in children and adolescents. Rheum Dis Clin North Am 21: 1115-37, 1995.

23. Maillard H, Szczesniak S, Martin L, et al.: Periartérite nouese cutanée: particularités diagnostiques et thérapeutiques de 9 cas. Ann Dermatol Venereol 126: 125-9, 1999.

24. Misago N, Mochizuki Y, Sekiyama-Kodera H, et al.: Cutaneous polyarteritis nodosa: therapy and clinical course in four cases. J Dermatol 28(12): 719-27, 2001. 